\title{
LA ANESTESIA LOCAL MEJORA SIGNIFICATIVAMENTE LA TOLERANCIA DE LA BIOPSIA PROSTÁTICA.
}

\author{
Javier Amalio Feltes Ochoa, Juan Passas Martínez, Nerea Felip Santamaría, Javier Romero \\ Otero, Alfredo Rodríguez Antolín y Oscar Leiva Galvis.
}

Servicio de Urología. Hospital Universitario 12 de Octubre. Madrid. España.

\begin{abstract}
Resumen.- La biopsia transrectal es uno de los procedimientos mas frecuentes en la práctica urológica. Usualmente las biopsias transrectales se han practicado sin administrarse anestesia, ante la consideración de una buena tolerancia. Sin embargo no es infrecuente encontrarnos ante pacientes con un nivel del dolor alto e incluso efectos adversos atribuibles al mismo.
\end{abstract}

OBJETIVO: Se evalúa en el presente trabajo si la anestesia local transrectal puede disminuir significativamente la percepción del dolor por el paciente.

MÉTODOS: Se incluyen en el siguiente estudio 131 pacientes consecutivos y sometidos a biopsia transrectal. Efectuándose la randomización 76 pacientes fueron biopsiados con anestesia y 55 representan el grupo control. Casos y controles no difieren en edad ni volumen prostático.
La anestesia se efectuó administrándose 10 cc del anestésico mepivacaína al 1\%, 5 cc en cada lado en el ángulo entre la próstata y las vesículas seminales. Se empleó una escala analógica y visual del dolor que se suministró al paciente una vez acabado el procedimiento.

RESULTADOS: En el grupo de pacientes anestesiados la media de valoración del dolor fue de 2,41 con una mediana de 2 y en el grupo control se obtuvo una media de 4,02 con una mediana de 4. Se realizó test de comparación de medias (t de Student), obteniéndose entre los grupos una diferencia estadísticamente significativa de 1,61 $1<0,00011$. Porcentualmente encontramos una diferencia en la escala del dolor de un $66 \%$ más en el grupo control.

CONCLUSIÓN: La utilización de anestesia en la realización de las biopsias transrectales disminuye significativamente la percepción del dolor por los pacientes. Este efecto junto a la tendencia en incrementar el número de biopsias, generalizará en poco tiempo el empleo de la anestesia local.

Palabras clave: Anestesia local. Tolerancia. Biopsia prostática. Ecografía Urológica.

Summary.- Transrectal biopsy is one of the most frequent procedures in urological practice. Generally, transrectal biopsies have been practiced without anesthesia, because of a supposed good tolerance. Nevertheless, it is not infrequent to find patients with a high level of pain and adverse effects attributable to such procedure.

OBJECTIVES: In the present article the effect of transrectal local anesthesia in order to significantly diminish the perception of pain by the patient is evaluated. 
METHODS: A total of 131 consecutive patients undergoing transrectal prostate biopsy are included in the study. After randomization, 76 patients were biopsiated with anesthesia and 55 represent the control group. Cases and control groups do not differ in age or prostate volume. Anesthesia consisted on a periprostatic nerve blockage with injection of $5 \mathrm{cc}$ of $1 \%$ mepivacaine solution in the angle between prostate and seminal vesicles bilaterally. A visual analogical scale for pain was used; it was given to the patient at the end of the procedure.

RESULTS: Mean pain value was 2.41 with a median of 2.0 in the group with anesthesia, and 4.02 with a median of 4 in the control group. A Student's t test comparing the means showed a statistically significant difference of $1.61(\mathrm{p}<0.0001)$. Pain in the scale was $66 \%$ greater in the control group.

CONCLUSION: The use of anesthesia in the performance of transrectal biopsies significantly diminishes the perception of pain by patients. This effect, along with the tendency to increase the number of biopsies, will result in short time in a more generalized use of local anesthesia.

Keywords: Local anesthesia. Tolerance. Prostatic biopsy. Urological ultrasound.

\section{INTRODUCCIÓN}

El cáncer de próstata (CP) es la neoplasia más frecuente en hombres y la segunda causa de muerte por cáncer en USA, después del cáncer de pulmón. En España ocupa el tercer puesto detrás del cáncer de pulmón y el colorrectal $(1,2)$.

La incidencia bruta del CP en la Comunidad de Madrid en el año 2000 fue de 100,4 casos por 100.000 hombres, con una incidencia ajustada a la población española de 120,1 casos/100.000 hombres y ajustada a la población mundial de 68,6 casos $\times 100.000(3)$.

La biopsia prostática guiada por Ecografía Transrectal (ETR) es el procedimiento de elección para el diagnóstico del CP en la actualidad (4). Al igual que otras pruebas diagnósticas invasivas, los pacientes presentan diversos grados de molestia o dolor durante y después del procedimiento (5). En algunos estudios se establece un $65 \%$ a $96 \%$ de pacientes con dolor de distinto grado durante la realiza- ción de esta técnica diagnóstica $(5,6)$. Otros refieren que un paciente de cada cinco se niega a una segunda biopsia sin algún tipo de anestesia (7). Incomodidad, ansiedad y dolor se desarrollan de forma proporcional al número de biopsias realizadas, cuyo número recientemente se ha incrementado con el fin de mejorar la efectividad del procedimiento. Actualmente la tendencia es efectuar un número de 8 o más biopsias por sesión; evidentemente el incremento del número de biopsias se refleja en mayor molestia por el paciente (8).

En base a estas evidencias y ante nuestra práctica diaria, hemos realizado un estudio prospectivo y randomizado, comparando la biopsia prostática bajo anestesia con inyección periprostática transrectal y la realización de esta prueba sin anestesia.

\section{MATERIAL Y MÉTODOS}

Selección de pacientes: Un total de 131 pacientes en edades comprendidas entre 46 y 79 años, fueron sometidos a biopsia guiada por ecografía transrectal (ETR), de forma consecutiva y divididos en dos grupos según una escala numérica aleatoria. El grupo 1 representan a los pacientes sometidos a anestesia (casos), y el grupo 2 corresponden a los controles, quienes no recibieron ningún tipo de anestesia ni placebo. Surgieron así 76 casos y 55 controles. La indicación de biopsia fueron: Niveles de PSA mayores de $4 \mathrm{ng} / \mathrm{dl}$ y/o tacto rectal anormal.

Todos los pacientes recibieron un enema la noche previa en su domicilio y la toma de Ciprofloxacino $500 \mathrm{mg}$ la mañana del procedimiento. No se administró ningún tipo de analgésicos previamente.

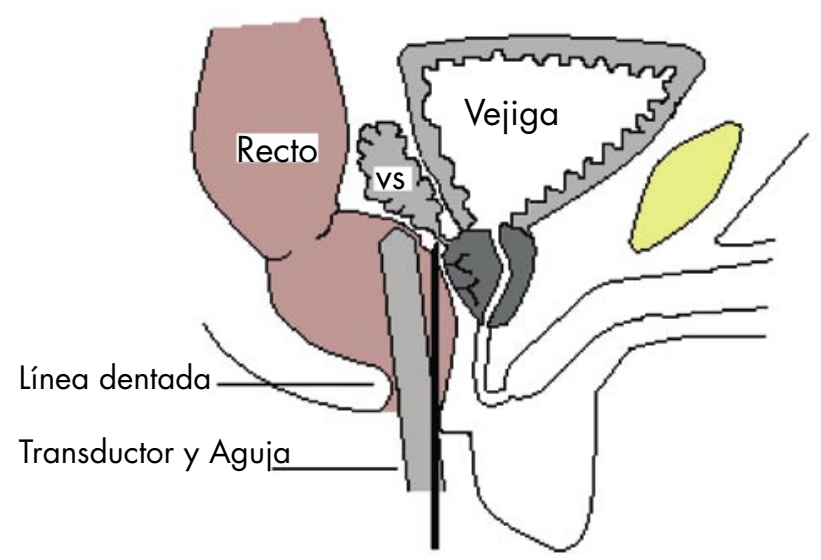

FIGURA 1. Introducción de la sonda del ecógrafo y punción en el ángulo vesiculoprostático. 

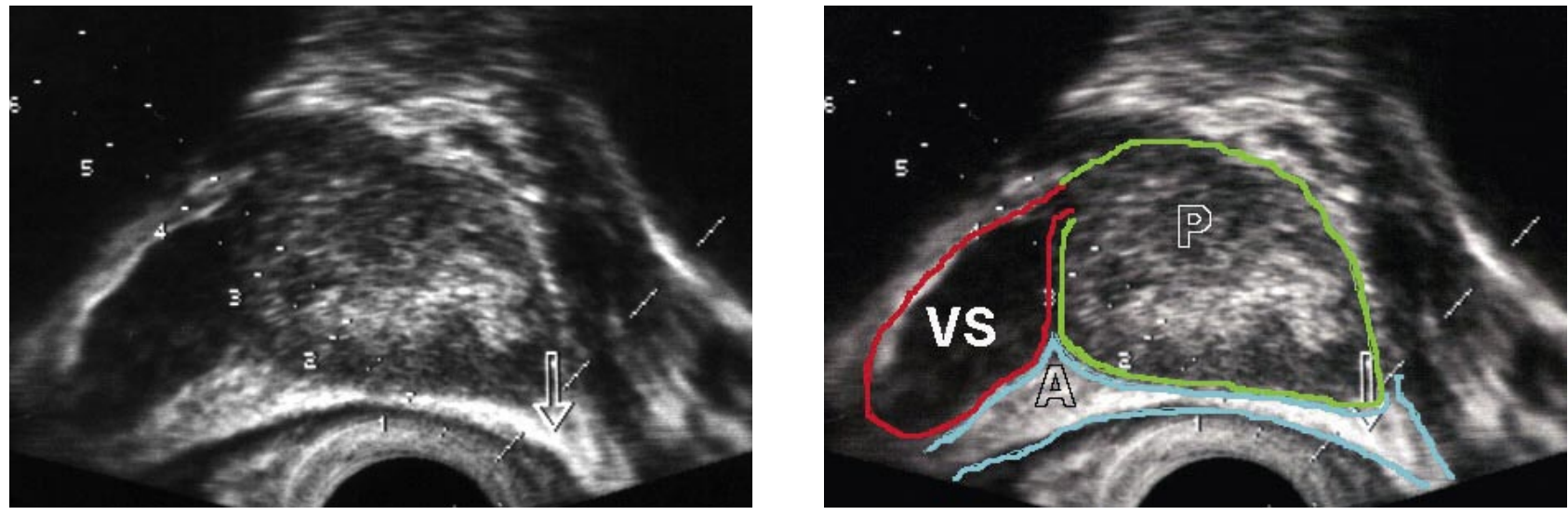

FIGURA 2. En la imagen ecográfica longitudinal se observa el ángulo (A), formado por la Próstata (P) y la Vesícula Seminal (VS) de aspecto similar a una montaña (Signo del Everest). Es una continuación ecográfica de la fascia de Denonvilliers (Flecha).

Procedimiento anestésico: Se utilizó $10 \mathrm{cc}$ de Mepivacaína al $1 \%$ que se inyectó a través de la misma aguja de la biopsia №18 de $20 \mathrm{~cm}$, distribuyendo $5 \mathrm{cc}$ en cada ángulo vesiculoprostático (AVP) (Figura 1), zona hiperecogénica triangular que se asemeja a una montaña (signo del Monte Everest) (Figura 2). Se observa tras la inyección, un habón que se traduce en una zona hipoecóica que diseca el espacio entre la próstata y el recto en la fascia de Denonvilliers (Figura 3).
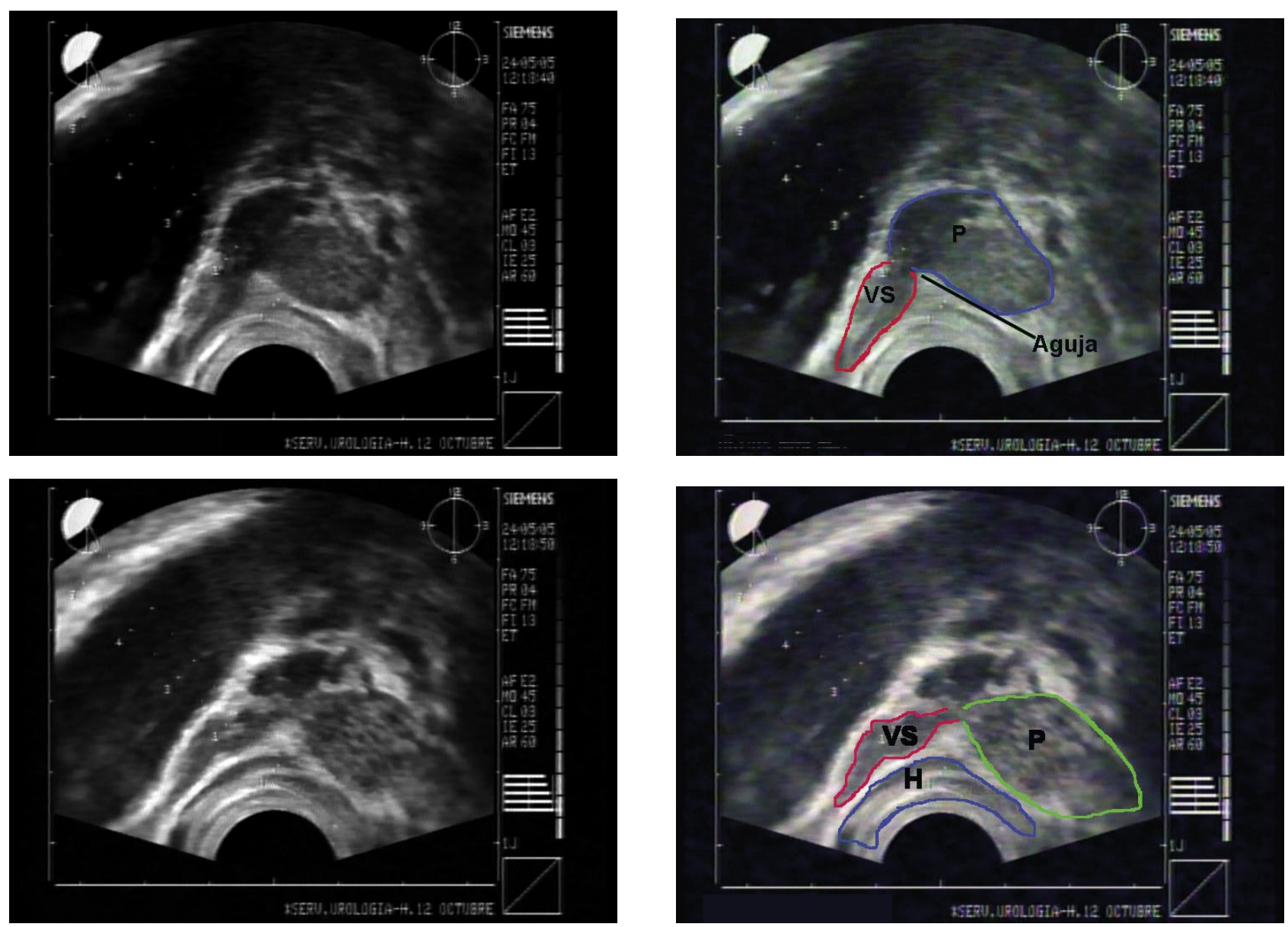

FIGURA 3. Inyección del anestésico en el ángulo vesiculoprostático, observándose posteriormente el habón (H). 
Tras 5 minutos de espera se procedió a la toma de biopsia con pistola automática, realizándose 10 tomas por sesión ( 3 mediosagitales y 2 laterales en cada lóbulo).

Posterior a la biopsia se entregó al paciente una hoja con una escala numérica y visual del dolor, en la cual cuantificaba la intensidad del dolor; dándole un valor 0 a la ausencia del dolor, 10 a la máxima sensación de dolor y valores intermedios a los distintos grados experimentados. También se registraron los efectos secundarios del procedimiento anestésico.

Análisis estadístico: Se establece para un nivel de confianza del $95 \%$ y un error relativo del $10 \%$, un tamaño muestral superior a 80 pacientes. Se analizaron variables de Edad, volumen prostático, biopsias con anestesia (casos) y sin ella (controles), valoración del dolor percibido por el paciente, complicaciones inmediatas del procedimiento anestésico y aquellas relacionadas a la misma biopsia. Comparación de las medias del dolor con la prueba t de Student.

\section{RESULTADOS}

Entre los 131 pacientes, 76 (58\%) biopsiados con anestesia y $55(42 \%)$ del grupo control, no

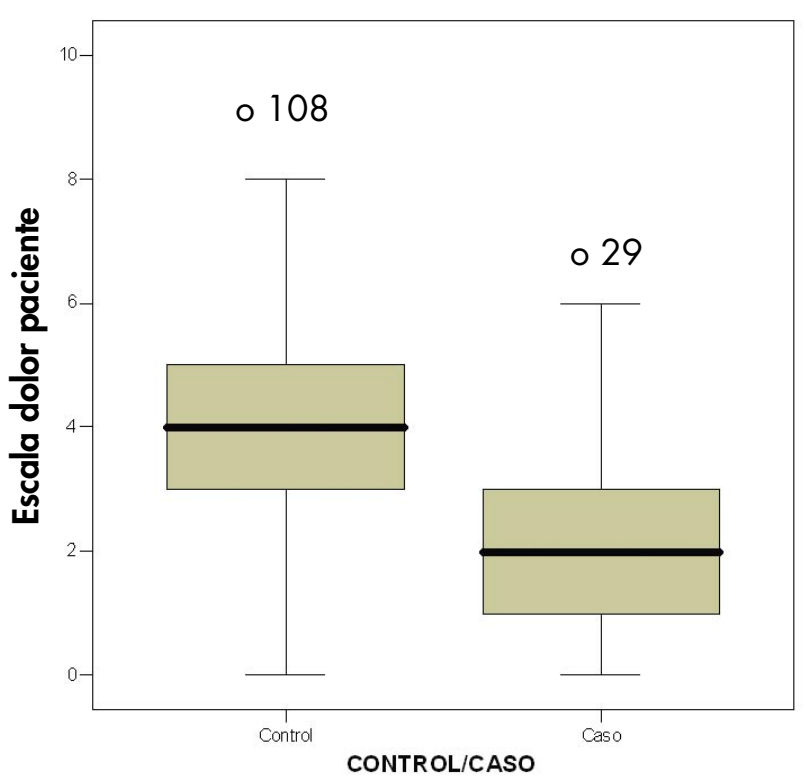

FIGURA 4. Comparación en la escala del dolor entre los dos grupos se obtuvieron diferencias representativas en las variables de edad, volumen prostático ni niveles de PSA.

No se observó ningún caso de reacción vasovagal en ninguno de los 131 pacientes ni diferencias significativas en los efectos secundarios relacionados al procedimiento como la rectorragia y la uretrorragia.

Obtuvimos una media del dolor de 2,41 para los casos y de 4,02 para los controles, con una mediana de 2,0 y 4,0 respectivamente (Figura 4). En la comparación de las medias del dolor se obtuvo una diferencia de 1,94 ( $p<0,0001)$. Porcentualmente se observa una diferencia en la escala del dolor de $66 \%$ entre ambos grupos. Si bien encontramos un discreto solapamiento en los valores intermedios, se registra valores extremos significativos para los controles (Figura 5).

Los pacientes que fueron sometidos previamente a biopsia sin anestesia manifestaban claramente su mejor tolerancia.

\section{COMENTARIOS}

Tradicionalmente se ha considerado al recto como una zona escasamente sensible debido a la pobre inervación por encima de la línea dentada. Este

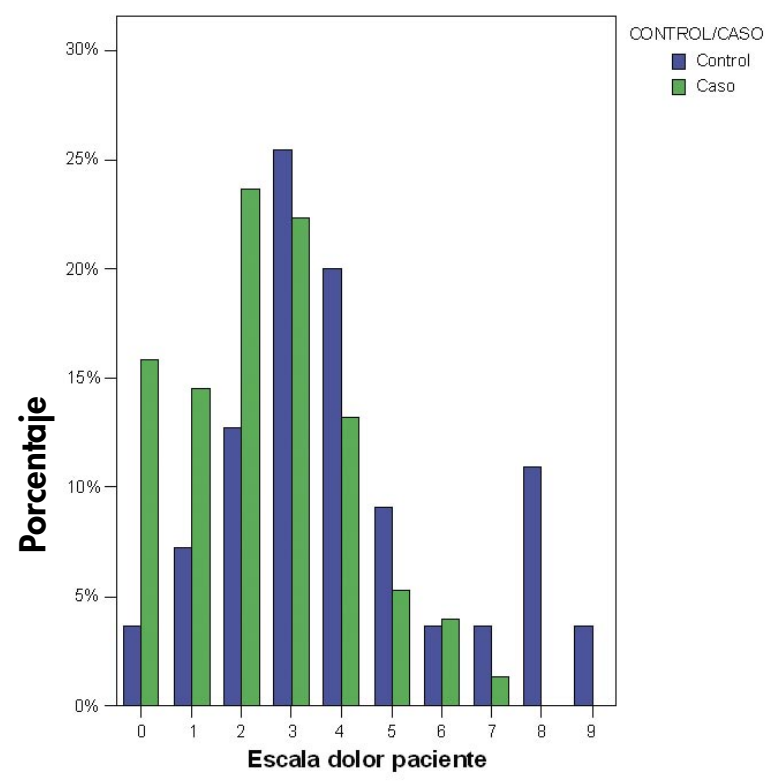

FIGURA 5. Distribución de los pacientes en la escala del dolor. 
hecho ha sido el fundamento de una gran mayoría de urólogos que siguen proponiendo las biopsias de próstata sin anestesia.

La inervación en la pelvis está representada por el plexo Hipogástrico inferior, el cual recibe aporte del plexo Hipogástrico superior. Del primero se origina el nervio Pélvico, que se encargará de transportar las fibras autonómicas y sensitivas para la próstata y otros órganos adyacentes (Figura 6). La inervación en el periné, sin embargo está representada por el nervio pudendo que se origina del plexo sacro (S2, S3 y S4), el cual otorgará ramas a la porción anal del recto (por debajo de la línea dentada), al esfínter estriado por debajo del diafragma urogenital, anastomosándose a las ramas terminales del $\mathrm{N}$. Pélvico y a toda la zona cutánea perineal (9).

Existen evidencias contrastadas que demuestran la necesidad de administrar al paciente alguna forma de anestesia durante la realización de esta prueba, principalmente porque mejora la percepción del dolor por el paciente y mejora la tolerancia a la misma $(10,11,12)$.

Se establece como desencadenantes de molestias y/o dolor, inicialmente la introducción de la sonda rectal, luego los movimientos de la misma en el recto durante el estudio, por último y mas representativo, la toma de biopsias.

La única manera de eliminar el dolor totalmente durante la biopsia transrectal, es bajo anestesia general, regional o sedación, especialmente en aquellas que requieran un alto número de biopsias y principalmente en las biopsias de saturación.

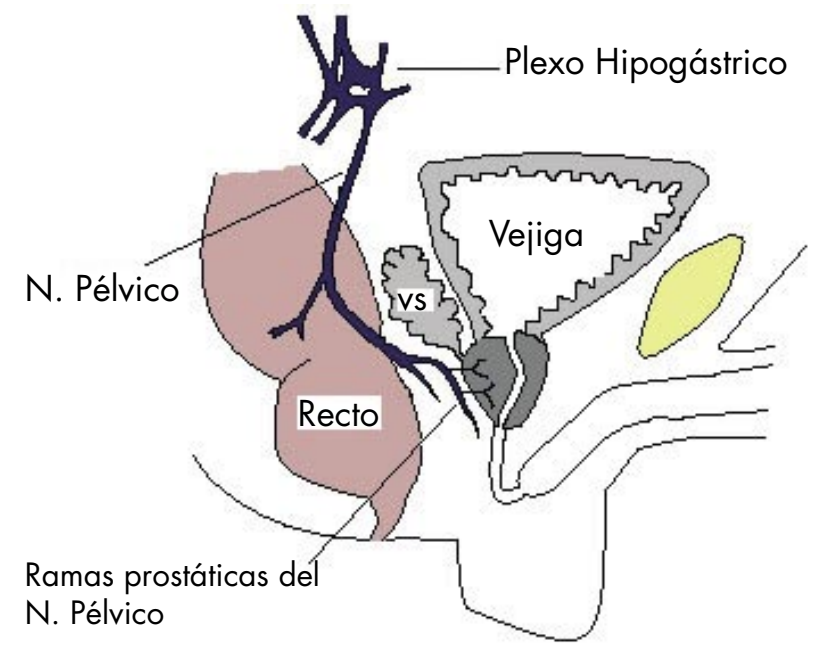

FIGURA 6. Esquema anatómico de la inervación de la próstata.
No se han difundido ampliamente estas técnicas por el costo, riesgo y complejidad de este tipo de procedimiento anestésico. Se han comunicado biopsias con inducción de agentes como el Oxido Nitroso (Entonox) y Propofol, con un margen de seguridad aceptable $(13,14)$.

Algunos abogan por la administración de analgésicos por vía venosa o sublingual momentos antes de procedimiento, sin dejar de ser éstos últimos claros métodos complementarios.

Dentro de los métodos locales se cita la instilación intrarrectal (IR) de gel con Lidocaína (GI) o Dimetil Sulfóxido (DMSO) con Lidocaína, utilizando como fundamento la gran capacidad de absorción de la mucosa rectal, difundiendo el anestésico hasta alcanzar los nervios periprostáticos. Existen trabajos que muestran resultados favorables con relación a esta forma de anestesia $(15,16)$; sin embargo, algunos como Desgrandchamps (17), Chang (18) y Cevik (19) no hallaron beneficios. En realidad, la absorción rectal es a través de los plexos hemorroidales que conducen a la circulación sistémica y no a la zona periprostática.

Existe otro método anestésico superior a la IR y es el bloqueo nervioso periprostático (BNP), al cual complementa y potencia $(6,20)$.

Mientras Wu y cols. (21) no encontraron diferencias significativas, hemos comprobado en nuestro estudio una clara disminución del dolor con el BNP como método anestésico aislado, hallando resultados similares a otros grupos de trabajos (Tabla I).

No encontramos diferencias significativas de las complicaciones como la hematuria, rectorragia o infecciones entre los dos grupos. No observamos ningún episodio de reacción vasovagal en toda la serie.

Obek y cols. $(20,22)$ han comunicado que con el BNP aumenta la incidencia de infecciones, debido probablemente a la adición de punciones extras y la pequeña colección tras la infiltración en un medio altamente colonizado como el recto. Mientras que disminuye la rectorragia en comparación a los controles. Otros no encuentran diferencias con respecto a estas complicaciones (23).

El anestésico más frecuentemente usado es la Lidocaína al $1 \%$, pero también se usan la Bupivacaína al $2,5 \%$ y la Articaína $1 \%$ y en nuestro caso la Mepivacaína al $1 \%$. Todos ellos con resultados y tolerancias similares $(24,25,26)$. 
Se puede inyectar el anestésico a nivel del AVP o base (27-31), la parte media lateroprostática, el ápex $(32,33)$ o combinando en varios sitios $(34$, 35). Taverna realiza una sola inyección de $10 \mathrm{ml}$ de anestésico en el ápex. Nosotros preferimos hacerlo en el AVP, por ser el sitio de llegada de la inervación a la próstata (Figura 6).
Algunos autores realizan una anestesia intraprostática (AIP), inyectando el anestésico en el interior de la próstata (36).

Es de interés reseñar que los grupos que han estudiado el efecto posterior encuentran que la biopsia con BNP no aumenta la dificultad de la conserva-

TABLA I. BIOPSIA PROSTÁTICA CON ANESTESIA POR BLOQUEO NERVIOSO PERIPROSTÁTICO (BNP).

\begin{tabular}{|c|c|c|c|c|c|}
\hline Referencia & $\begin{array}{c}\text { № } \\
\text { Pacientes }\end{array}$ & $\begin{array}{l}\text { Comparación } \\
\text { (№ Ptes) }\end{array}$ & $\begin{array}{l}\text { Volumen anestési- } \\
\text { co inyectado }(\mathrm{ml})^{*}\end{array}$ & Escala del dolor & $\begin{array}{l}\text { Método de } \\
\text { inyección }\end{array}$ \\
\hline $\begin{array}{l}\text { Nash (11) } \\
1996\end{array}$ & 64 & $\begin{array}{l}5 \mathrm{ml} \mathrm{L} 1 \%(34) \\
5 \mathrm{ml} \mathrm{SS}(30)\end{array}$ & 5 & $\begin{array}{l}1,6 \text { vs } 2,4 \\
2,9 \text { vs } 3,0\end{array}$ & $\begin{array}{l}\text { Solo a un lado de la } \\
\text { próstata (I) }\end{array}$ \\
\hline $\begin{array}{l}\text { Soloway (12) } \\
2000\end{array}$ & 50 & $5 \mathrm{ml} \mathrm{L1 \% (50)}$ & 10 & & $\mathrm{~B}(\mathrm{II}), \mathrm{M}(\mathrm{II})$ y $\mathrm{A}(\mathrm{II})$ \\
\hline $\begin{array}{l}\text { Seymour (32) } \\
2001 \\
\end{array}$ & 157 & 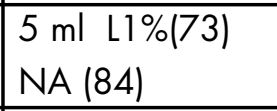 & 10 & $\begin{array}{l}1,95 \pm 0,65 \\
1,53 \pm 0,7\end{array}$ & $A(I I)$ \\
\hline \begin{tabular}{|l} 
Pareek (27) \\
2001
\end{tabular} & 132 & $\begin{array}{l}2,5 \mathrm{ml} \mathrm{L1} \%(66) \\
2,5 \mathrm{ml} \mathrm{SS}(66)\end{array}$ & 10 & $\begin{array}{l}2,7 \pm 0,21 \\
4,7 \pm 0,26\end{array}$ & $\mathrm{~B}(\mathrm{II})$ \\
\hline $\begin{array}{l}\text { Schostak (35) } \\
2002\end{array}$ & 170 & 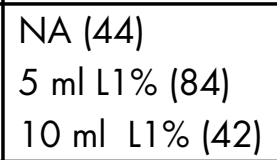 & $\begin{array}{l}10 \\
20\end{array}$ & $\begin{array}{l}2,33 \\
1,68-1,07 \\
1,23\end{array}$ & $\begin{array}{l}\text { G2(44) } 5 \mathrm{ml} \mathrm{c} / M(I I) \\
\text { G3(40) } 5 \mathrm{ml} \mathrm{c} / A(I I) \\
\text { G4(42) c/A y } M(I I)\end{array}$ \\
\hline $\begin{array}{l}\text { Taverna (26) } \\
2002\end{array}$ & 100 & $10 \mathrm{ml} \mathrm{L1 \%}$ & 10 & $\begin{array}{l}93 \% \text { Score } 1-3 \\
7 \% \text { Score }>4\end{array}$ & $\mathrm{~A}(\mathrm{I})$ \\
\hline $\begin{array}{l}\text { Bulbul (28) } \\
2002\end{array}$ & 72 & $\begin{array}{l}2 \mathrm{ml} \mathrm{L2 \%} \mathrm{(47)} \\
\text { NA (25) }\end{array}$ & 4 & $\begin{array}{l}11 \% \text { Dolor } M-S \\
32 \% \text { Dolor } M-S\end{array}$ & $\mathrm{~B}(\mathrm{II})$ \\
\hline $\begin{array}{l}\text { Kaver (34) } \\
2002\end{array}$ & 152 & 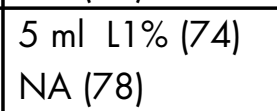 & 15 & $\begin{array}{l}1,8-1,2 A-B \\
5,6-4,1 \text { A - B }\end{array}$ & $B(I I)$ y $A(I)$ \\
\hline $\begin{array}{l}\text { Leibovici (29) } \\
2002\end{array}$ & 90 & $\begin{array}{l}5 \mathrm{ml} \mathrm{L1} \%(45) \\
5 \mathrm{ml} \mathrm{SS} \mathrm{(45)}\end{array}$ & 10 & $\begin{array}{l}1,5 \\
3,98\end{array}$ & $\mathrm{~B}(\mathrm{II})$ \\
\hline \begin{tabular}{|l|} 
Berger (30) \\
2003 \\
\end{tabular} & 100 & 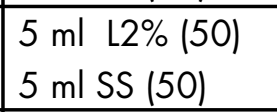 & 10 & $\begin{array}{l}3,62 \\
0,76\end{array}$ & $\mathrm{~B}(\mathrm{II})$ \\
\hline \begin{tabular}{|l} 
Jones (37) \\
2003 \\
\end{tabular} & 60 & $\begin{array}{l}5 \mathrm{ml} \mathrm{L} 1 \% \\
\mathrm{NA}\end{array}$ & 10 & $\begin{array}{l}1,4 \\
4,5 \\
\end{array}$ & $\mathrm{~B}(\mathrm{II})$ \\
\hline $\begin{array}{l}\text { Ozden (23) } \\
2003\end{array}$ & 175 & $\begin{array}{l}5 \mathrm{ml} \mathrm{SS} \mathrm{(25)} \\
2,5 \mathrm{ml} \mathrm{L} 1 \%(50) \\
5 \mathrm{ml} \mathrm{L} 1 \%(50) \\
10 \mathrm{ml} \mathrm{L} 1 \%(50)\end{array}$ & $\begin{array}{l}\text { G2 - G3: } 2,5 \\
\text { G4 - G5: } 5 \\
\text { G6 - G7: } 10\end{array}$ & $\begin{array}{l}\text { G1 4,0 } \\
\text { G2 3,0 } \\
\text { G3 - G5 2,0 } \\
\text { G6 - G7 1,0 }\end{array}$ & $\begin{array}{l}\text { G1 SS B(I) } \\
\text { G2,G4 y G6 B(II) } \\
\text { G3,G5 y G7 B+A(II) }\end{array}$ \\
\hline $\begin{array}{l}\text { Inal (3 1) } \\
2004\end{array}$ & 90 & $\begin{array}{l}\text { NA (30) } \\
3 \mathrm{ml} \mathrm{SS} \mathrm{(30)} \\
3 \mathrm{ml} \mathrm{L1 \% (30)}\end{array}$ & 6 & $\begin{array}{l}5,65 \pm 2,35 \\
6,25 \pm 2,04 \\
3,16 \pm 2,14\end{array}$ & $\mathrm{~B}$ (II) \\
\hline
\end{tabular}

L: Lidocaína. NA: No recibió Anestesia. SS: Suero salino. B: Base prostática o ángulo vesiculoprostático. M: Porción mediolateral prostática. A: Ápex prostático. (II): Inyección unilateral. (II): Inyección bilateral. * Volumen anestésico total empleado. 
ción de bandeletas en la cirugía radical de próstata $(37,38)$.

\section{CONCLUSIÓN}

Creemos firmemente que la anestesia por BNP es un método válido, fácil de realizar de forma aislada o combinando con otros métodos (Gel intrarrectal, analgésicos orales o intravenosos). Permite una mejor tolerancia de los pacientes a las molestias generadas durante las biopsias transrectales, además de la posibilidad de obtener un número igual o mayor de 10 muestras en caso necesario sin incrementar la percepción dolorosa y aportando mayor aceptación entre aquellos pacientes que requerirán una segunda biopsia.

\section{BIBLIOGRAFÍA y LECTURAS RECOMENDADAS (*lectura de interés $y$ ** lectura fundamental)}

1. WORLD HEALTH ORGANIZATION: "Cancer incidence, mortality and prevalence worldwide, 2000 estimates". http://www.who.int/health-topics/cancer/en.

2. FARKAS, A.; SCHNEIDER, D.; PERROTTI, M. y cols.: "National trends in the epidemiology of prostate cancer, 1973 to 1994: evidence for the effectiveness of prostate-specific antigen screening". Urology, 52: 444, 1998.

3. HERRANZ, F.: "El cáncer de próstata en la Comunidad de Madrid en el año 2000". Ed. AstraZeneca.

4. COLEY, C.M.; BARRY, M.J.; FLEMING, C. y cols.: "Should Medicare provide reimbursement for prostate-specific antigen testing for early detection of prostate cancer? Part II: early detection strategies". Urology, 46: 125, 1995.

5. ZISMAN, A.; LEIBOVICI, D.; KLEINMANN, J. y cols.: "The impact of prostate biopsy on patient well-being: a prospective study of pain, anxiety and erectile dysfunction". J. Urol., 165: 445, 2001.

6. RODRÍGUEZ, A.; KIRIAKOU, G.; LERAY, E. y cols.: "Prospective study comparing two methods of anaesthesia for prostate biopsias: Apex periprostatic nerve block versus intrarectal lidocaine gel: Review of the literature”. Eur. Urol., 44: 195, 2003.

7. IRANI, J.; FOURNIER, F.; BON, D. y cols.: "Patient tolerance of transrectal ultrasound-guided biopsy of prostate". Br. J. Urol., 79: 608, 1997.
8. NAUGHTON, C.K.; ORNSTEIN, D.K.; SMITH, D.S. y cols.: "Pain and morbidity of transrectal ultrasound guided prostate biopsy: A prospective randomized trial of 6 versus 12 cores". J. Urol., 163: 168, 2000.

*9. HOLLABAUGH, R.S. Jr.; DMOCHOWSKI, R.R.; STEINER, M.S.: "Neuroanatomy of the male rhabdosphincter". Urology, 49: 426, 1997.

**10. AUTORINO, R.; DE SIO, M.; DI LORENZO, G. y cols.: "How to decrease pain during transrectal ultrasound guided prostate biopsy: a look at the literature". J. Urol., 174: 2091, 2005.

*11. NASH, P.A.; BRUCE, J.E.; INDUDHARA, R. y cols.: "Transrectal Ultrasound guided prostatic nerve blockade eases systematic needle biopsy of the prostate". J. Urol., 155: 607, 1996.

*12. SOLOWAY, M.S.; OBEK, C.: "Periprostatic local anesthesia before ultrasound guided prostate biopsy". J. Urol., 163: 172, 2000.

13. MASOOD, J.; SHAH, N.; LANE, T. y cols.: "Nitrous oxide (Entonox) inhalation and tolerance of transrectal ultrasound guided prostate biopsy: a double-blind randomized controlled study". J. Urol., 168: 116, 2002.

14. PETERS, J.L.; THOMPSON, A.C.; McNICHOLAS, T.A. y cols.: "Increased patient satisfaction from transrectal ultrasonography and biopsy under sedation". BJU Int., 87: 827, 2001.

*15. ISSA, M.M.; BUX, S.; CHUN, T. y cols.: "A randomized prospective trial of intrarectal lidocaine for pain control during transrectal prostate biopsy: the Emory University experience". J. Urol., 164: 397, 2000.

16. KRAVCHICK, S.; PELED, R.; BEN-DOR,D. y cols.: "Comparison of different local anesthesia techniques during TRUS-guided biopsies: a prospective pilot study". Urology, 65: 109, 2005.

*17. DESGRANDCHAMPS, F.; MERIA, P.; IRANI, J. y cols.: "The rectal administration of lidocaine gel and tolerance of transrectal ultrasonographyguided biopsy of the prostate: a prospective randomized placebo-controlled study”. BJU Int., 83: 1007, 1999.

18. CHANG, S.S.; ALBERTS, G.; WELLS, N. y cols.: "Intrarectal lidocaine during transrectal prostate biopsy: results of a prospective double-blind randomized trial". J. Urol., 166: 2178, 2001.

19. CEVIK, I.; OZVERI, H.; DILLIOGLUGIL, O. y cols.: "Transrectal periprostatic lidocaine injection anesthesia for transrectal prostate biopsy: a prospective study". Prostate Cancer Prostatic Dis., 6: 311, 2003.

*20. OBEK, C.; OZKAN, B.; TUNC, B. y cols.: "Comparison of 3 different methods of anesthesia before transrectal prostate biopsy: a prospective randomized trial". J. Urol., 172: 502, 2004. 
*21. WU, C.L.; CARTER, H.B.; NAQIBUDDIN, M. y cols.: "Effect of local anesthetics on patient recovery after transrectal biopsy". Urology, 57: 925, 2001.

22. OBEK, C.; ONAL, B.; OZKAN, B. y cols.: "Is periprostatic local anesthesia for transrectal ultrasound guided prostate biopsy associated with increased infectious or hemorrhagic complications? A prospective randomized trial". J. Urol., 168: 558, 2002.

23. OZDEN, E.; YAMAN, O.; GOGUS, C. y cols.: "The optimum doses of and injection locations for periprostatic nerve blockade for transrectal ultrasound guided biopsy of the prostate: a prospective, randomized, placebo controlled study". J. Urol., 170: 2319, 2003.

24. LEE-ELLIOTT, C.E.; DUNDAS, D.; PATEL, U.: "Randomized trial of lidocaine vs lidocaine/bupivacaine periprostatic injection on longitudinal pain scores after prostate biopsy". J. Urol., 171: 247, 2004.

*25. RABETS, J.C.; JONES, J.S.; PATEL, A.R. y cols.: "Bupivacaine provides rapid, effective periprostatic anaesthesia for transrectal prostate biopsy". BJU Int., 93: 1216, 2004.

26. VON KNOBLOCH, R.; WEBER, J.; VARGA, Z. y cols.: "Bilateral fine-needle administered local anaesthetic nerve block for pain control during TRUS-guided multi-core prostate biopsy: a prospective randomised trial". Eur. Urol., 41: 508, 2002.

27. PAREEK, G.; ARMENAKAS, N.A.; FRACCHIA, J.A.: "Periprostatic nerve blockade for transrectal ultrasound guided biopsy of the prostate: a randomized double-blind, placebo controlled study". J. Urol., 166: 894, 2001.

28. BULBUL, M.A.; HADDAD, M.C.; KHAULI, R.B. y cols.: "Periprostatic infiltration with local anaesthesia during transrectal ultrasound-guided prostate biopsy is safe, simple, and effective: a pilot study". Clin. Imaging, 26: 129, 2002.

29. LEIBOVICI, D.; ZISMAN, A.; SIEGEL, Y.I. y cols.: "Local anesthesia for prostate biopsy by pe- riprostatic lidocaine injection: a double-blind placebo controlled study". J. Urol., 167: 563, 2002.

30. BERGER, A.P.; FRAUSCHER, F.; HALPERN, E.J. y cols.: "Periprostatic administration of local anesthesia during transrectal ultrasound-guided biopsy of the prostate: a randomized double-blind placebo-controlled study". Urology, 61: 585, 2003.

31. INAL, G.; YAZICI, S.; ADSAN, O. y cols.: "Effect of periprostatic nerve blockade before transrectal ultrasound-guided prostate biopsy on patient comfort: a randomized placebo controlled study". Int. J. Urol., 11: 148, 2004.

32. SEYMOUR, H.; PERRY, M.J.; LEE.ELLIOTT, C. y cols.: "Pain after transrectal ultrasonography-guided prostate biopsy: the advantages of periprostatic local anaesthesia". BJU Int., 88: 540, 2001.

*33. TAVERNA, G.; MAFFEZZINI, M.; BENETTI, A. y cols.: "A single injection of lidocaine as local anesthesia for ultrasound guided needle biopsy of the prostate". J. Urol., 167: 222, 2002.

34. KAVER, I.; NICOLA, J.; MATZKIN, H. y cols.: "Randomized prospective study of periprostatic local anaesthesia during transrectal ultrasoundguided prostate biopsy". Urology, 59: 405, 2002.

35. SCHOSTAK, M.; CHRISTOPH, F.; MULLER, M. y cols.: "Optimizing local anaesthesia during 10-core biopsy of the prostate". Urology, 60: 253, 2002.

36. MUTAGUCHI, K.; SHINOHARA, K.; MATSUBARA, A. y cols.: "Local anesthesia during 10 core biopsy of the prostate: Comparison of 2 methods". J. Urol., 173: 742, 2005.

37. JONES, J.S.; ULCHAKER, J.; NELSON, D. y cols.: "Periprostatic local anesthesia eliminates pain of office-based transrectal prostate biopsy". Prostate Cancer Prostatic Dis., 6: 53, 2003.

38. VAIDYA, A.; SOLOWAY, M.S.: "Periprostatic local anesthesia before ultrasound-guided prostate biopsy: an update of the Miami experience". Eur. Urol., 40: 135, 2001. 